\title{
Inferring a level-1 phylogenetic network from a dense set of rooted triplets ${ }^{2}$
}

\author{
Jesper Jansson $^{\mathrm{a}, *}$, Wing-Kin Sung ${ }^{\mathrm{a}, \mathrm{b}, *}$

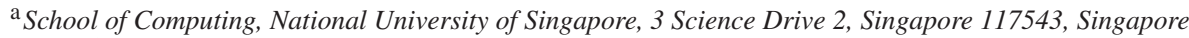 \\ ${ }^{\mathrm{b}}$ Genome Institute of Singapore, 60 Biopolis Street, Genome, Singapore 138672, Singapore
}

\begin{abstract}
We consider the following problem: Given a set $\mathscr{T}$ of rooted triplets with leaf set $L$, determine whether there exists a phylogenetic network consistent with $\mathscr{T}$, and if so, construct one. We show that if no restrictions are placed on the hybrid nodes in the solution, the problem is trivially solved in polynomial time by a simple sorting network-based construction. For the more interesting (and biologically more motivated) case where the solution is required to be a level-1 phylogenetic network, we present an algorithm solving the problem in $\mathrm{O}\left(|\mathscr{T}|^{2}\right)$ time when $\mathscr{T}$ is dense, i.e., when $\mathscr{T}$ contains at least one rooted triplet for each cardinality three subset of $L$. We also give an $\mathrm{O}\left(|\mathscr{T}|^{5 / 3}\right)$-time algorithm for finding the set of all phylogenetic networks having a single hybrid node attached to exactly one leaf (and having no other hybrid nodes) that are consistent with a given dense set of rooted triplets.
\end{abstract}

(C) 2006 Elsevier B.V. All rights reserved.

Keywords: Phylogenetic network construction; Rooted triplet; Sorting network; Algorithm

\section{Introduction}

A phylogenetic network is a generalization of a phylogenetic tree in which internal nodes are allowed to have more than one parent. Phylogenetic networks are used to represent evolutionary relationships that cannot be adequately described in a single tree structure due to evolutionary events such as recombination, horizontal gene transfer, or hybrid speciation which imply convergence between objects [10,11,20,21,23].

Several methods for constructing and for comparing phylogenetic networks have been proposed recently $[4,6,10,19-21,23]$. In this paper, we consider the problem of constructing a phylogenetic network from a set of rooted triplets (see below for a formal problem definition). In particular, we assume that the input forms a dense set, meaning that the input contains at least one rooted triplet for each cardinality three subset of the objects being studied, and that the underlying phylogenetic network is a level-1 network, meaning that each biconnected component in the undirected version of the network induces a subgraph in the directed version of the network that has at most one node with two parents. The biological significance of level-1 phylogenetic networks, there referred to as galled-trees, is discussed in [10]. The rationale for assuming the input to consist of rooted triplets is that although computationally expensive

\footnotetext{
t5 A preliminary version of this article appeared in Proceedings of the Tenth Annual International Computing and Combinatorics Conference (COCOON 2004), volume 3106 of Lecture Notes in Computer Science, pp. 462-471, Springer, 2004.

* Corresponding authors. School of Computing, National University of Singapore, 3 Science Drive 2, Singapore 117543, Singapore.

E-mail addresses: jansson@ comp.nus.edu.sg, jj@df.lth.se (J. Jansson), ksung@comp.nus.edu.sg (W.-K. Sung).
} 


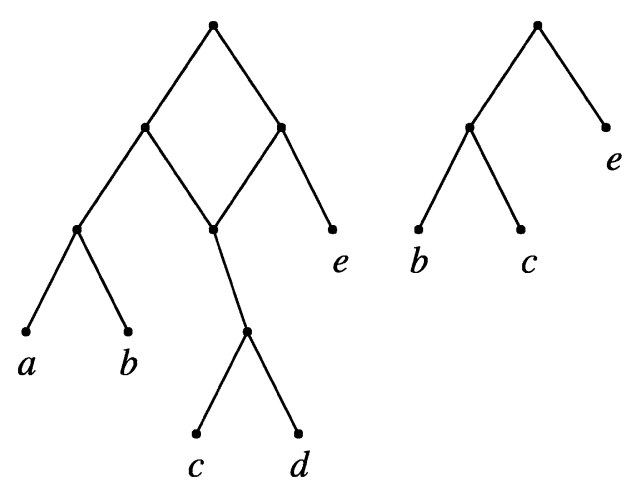

Fig. 1. Let $N$ be the level-1 phylogenetic network on the left. The rooted triplet $(\{b, c\}, e)$ shown on the right is consistent with $N$. Note that $(\{c, e\}, b)$ is also consistent with $N$.

methods for constructing reliable phylogenetic trees such as maximum likelihood are infeasible for large sets of objects, they can be applied to infer highly accurate trees for smaller, overlapping subsets of the objects (see, e.g., [5]). One may thus apply maximum likelihood to each cardinality three subset $L^{\prime}$ of the objects and then select the most likely rooted triplet for $L^{\prime}$ to get a dense input set. ${ }^{1}$ Moreover, in some applications, the data obtained experimentally may already have the form of rooted triplets; for example, Sibley-Ahlquist-style DNA-DNA hybridization experiments (see [17]) can yield rooted triplets directly.

\subsection{Definitions}

A rooted triplet is a binary, rooted, unordered tree with three distinctly labeled leaves. The unique rooted triplet on leaf set $\{x, y, z\}$ in which the lowest common ancestor of $x$ and $y$ is a proper descendant of the lowest common ancestor of $x$ and $z$ (or equivalently, where the lowest common ancestor of $x$ and $y$ is a proper descendant of the lowest common ancestor of $y$ and $z)$ is denoted by $(\{x, y\}, z)$. A set $\mathcal{T}$ of rooted triplets is called dense if for each $\{x, y, z\} \subseteq L$, where $L$ is the set of all leaves occurring in $\mathcal{T}$, at least one of $(\{x, y\}, z),(\{x, z\}, y)$, and $(\{y, z\}, x)$ belongs to $\mathcal{T}$.

A phylogenetic network is a connected, rooted, simple, directed acyclic graph in which: (1) each node has outdegree at most 2; (2) each node has indegree 1 or 2, except the root node which has indegree 0 ; (3) no node has both indegree 1 and outdegree 1; and (4) all nodes with outdegree 0 are labeled in such a way that no two nodes are assigned the same label. From here on, nodes of outdegree 0 are referred to as leaves and identified with their corresponding elements in $L$. We refer to nodes with indegree 2 as hybrid nodes.

For any phylogenetic network $N$, let $\mathcal{U}(N)$ be the undirected graph obtained from $N$ by replacing each directed edge by an undirected edge. $N$ is called a level-f phylogenetic network if, for every biconnected component $B$ in $\mathcal{U}(N)$, the subgraph of $N$ induced by the set of nodes in $B$ contains at most $f$ nodes with indegree 2 . Note that if $f=0$ then $N$ is a tree.

We denote the set of leaves in a rooted triplet $t$ or a phylogenetic network $N$ by $\Lambda(t)$ or $\Lambda(N)$, respectively. A rooted triplet $t$ is consistent with the phylogenetic network $N$ if $t$ is an embedded subtree of $N$. See Fig. 1 for an example. A set $\mathcal{T}$ of rooted triplets is consistent with $N$ if every $t_{i} \in \mathcal{T}$ is consistent with $N$.

The problem we focus on in this paper is: given a set $\mathcal{T}=\left\{t_{1}, \ldots, t_{k}\right\}$ of rooted triplets, construct a level-1 phylogenetic network $N$ with $\Lambda(N)=\bigcup_{t_{i} \in \mathcal{T}} \Lambda\left(t_{i}\right)$ such that $\mathcal{T}$ is consistent with $N$, if such a network exists; otherwise, output null. Throughout this paper, we let $L$ represent the leaf set $\bigcup_{t_{i} \in \mathcal{T}} \Lambda\left(t_{i}\right)$ in the problem definition above, and we write $n=|L|$ and $k=|\mathcal{T}|$. Note that if the input is dense then $\left(\begin{array}{l}n \\ 3\end{array}\right) \leqslant k \leqslant 3 \cdot\left(\begin{array}{l}n \\ 3\end{array}\right)$, i.e., $k=\Theta\left(n^{3}\right)$.

Finally, for any set $\mathcal{T}$ of rooted triplets and $L^{\prime} \subseteq L$, we define $\mathcal{T} \mid L^{\prime}$ as the subset of $\mathcal{T}$ consisting of all rooted triplets $(\{x, y\}, z)$ with $\{x, y, z\} \subseteq L^{\prime}$.

\footnotetext{
${ }^{1}$ A similar approach is used in the quartet method paradigm $[16,18]$ for reconstructing unrooted phylogenetic trees: first infer the unrooted topology of each cardinality four subset of the leaf set to obtain a complete set of quartets (unrooted, distinctly leaf-labeled trees each having four leaves and no nodes of degree two), then combine the quartets into an unrooted tree.
} 


\subsection{Related work}

Aho et al. [1] presented an $\mathrm{O}(k n)$-time algorithm for determining whether a given set of $k$ rooted triplets on $n$ leaves is consistent with some rooted, distinctly leaf-labeled tree (i.e., a level-0 phylogenetic network), and if so, returning such a tree. Several years later, Henzinger et al. [12] showed how to implement the algorithm of Aho et al. to run in $\min \left\{\mathrm{O}\left(k n^{0.5}\right), \mathrm{O}\left(k+n^{2} \log n\right)\right\}$ time. Gassieniec et al. [8] considered a version of the problem where the leaves in the output tree are required to comply with a left-to-right leaf ordering given as part of the input. Related optimization problems where the objective is to construct a rooted tree consistent with the maximum number of rooted triplets in the input or to find a maximum cardinality subset $L^{\prime}$ of $L$ such that $\mathcal{T} \mid L^{\prime}$ is consistent with some tree have been studied in $[3,8,9,14,24,15]$ and in Jansson [15], respectively.

The analog of the problem considered by Aho et al. for unrooted trees is NP-hard, even if all of the input trees are quartets [22]. Fortunately, certain useful optimization problems involving quartets can be approximated efficiently [16,18]. For a survey on quartet-based methods for inferring unrooted phylogenetic trees and related computational complexity results, see [18].

Nakhleh et al. [20] gave an algorithm for reconstructing a level-1 phylogenetic network from two distinctly leaflabeled, binary, rooted, unordered trees with identical leaf sets. It runs in time which is polynomial in the number of leaves and the number of hybrid nodes in the underlying phylogenetic network. They also considered the case where the two input trees may contain errors but where only one hybrid node is allowed.

We remark that the deterministic algorithm for dynamic graph connectivity employed in the algorithm of Henzinger et al. [12] mentioned above can in fact be replaced with a more recent one due to Holm et al. [13] to yield the following improvement.

Lemma 1 (Jansson et al. [15]). The algorithm of Aho et al. can be implemented to run in $\min \left\{\mathrm{O}\left(k \log ^{2} n\right), \mathrm{O}(k+\right.$ $\left.\left.n^{2} \log n\right)\right\}$ time.

\subsection{Our results and organization of the paper}

We observe that if no restriction is placed on the level of the phylogenetic network, then the problem can be trivially solved using a sorting network-based construction in Section 2. Next, in Section 3, we present an $\mathrm{O}\left(n^{5}\right)$-time algorithm called OneHybridLeaf for inferring the set of all phylogenetic networks with one hybrid node to which exactly one leaf is attached that are consistent with a given dense set $\mathcal{T}$ of rooted triplets. This algorithm is subsequently used in Section 4, where we give a more general algorithm called LevelOne for constructing a level-1 phylogenetic network consistent with $\mathcal{T}$ (if one exists) in $\mathrm{O}\left(n^{6}\right)$ time when $\mathcal{T}$ is dense. (Since $k=|\mathcal{T}|=\Theta\left(n^{3}\right)$ when $\mathcal{T}$ is dense, the running times of OneHybridLeaf and LevelOne can also be expressed as $\mathrm{O}\left(k^{5 / 3}\right)$ or $\mathrm{O}\left(|\mathcal{T}|^{5 / 3}\right)$, and $\mathrm{O}\left(k^{2}\right)$ or $\mathrm{O}\left(|\mathcal{T}|^{2}\right)$, respectively.)

\section{Constructing an unrestricted phylogenetic network}

Given any set $\mathcal{T}$ of rooted triplets with a leaf set $L$, we can always construct a level-f phylogenetic network $N$ where $f$ is unrestricted such that $N$ is consistent with $\mathcal{T}$. Moreover, the construction can be carried out in time which is polynomial in the size of $\mathcal{T}$ as follows. Let $P$ be any sorting network (see, e.g., [7]) for $n$ elements with a polynomial number $p$ of comparator stages. Build a directed acyclic graph $Q$ from $P$ with $(p+2) \cdot n$ nodes $\left\{Q_{i, j} \mid 0 \leqslant i \leqslant p+\right.$ $1,1 \leqslant j \leqslant n\}$ such that there is a directed edge $\left(Q_{i, j}, Q_{i+1, j}\right)$ for every $0 \leqslant i \leqslant p$ and $1 \leqslant j \leqslant n$, and two directed edges $\left(Q_{i, j}, Q_{i+1, k}\right)$ and $\left(Q_{i, k}, Q_{i+1, j}\right)$ for every comparator $(j, k)$ at stage $i$ in $P$ for $1 \leqslant i \leqslant p$. Then, for $1 \leqslant j \leqslant n-1$, add the directed edge $\left(Q_{0, j}, Q_{0, j+1}\right)$. See Fig. 2 . Finally, distinctly label the nodes $\left\{Q_{p+1, j} \mid 1 \leqslant j \leqslant n\right\}$ by $L$, and for each node in $Q$ having indegree 1 and outdegree 1 (if any), contract its outgoing edge to obtain $N$.

Lemma 2. For any $\{x, y, z\} \subseteq L$, all three of $(\{x, y\}, z),(\{x, z\}, y)$, and $(\{y, z\}, x)$ are consistent with $N$.

Proof. Assume without loss of generality that $L=\{1,2, \ldots, n\}$. Since $P$ is a sorting network, there are $n$ disjoint paths in $Q$ from $\left(Q_{0, \pi(1)}, Q_{0, \pi(2)}, \ldots, Q_{0, \pi(n)}\right)$ to $\left(Q_{p+1,1}, Q_{p+1,2}, \ldots, Q_{p+1, n}\right)$ for any given permutation $\pi$ of $\{1,2, \ldots, n\}$; in particular, this holds for any permutation $\pi_{x}$ such that $\pi_{x}(x)=1, \pi_{x}(y)=2$, and $\pi_{x}(z)=3$. Therefore, 


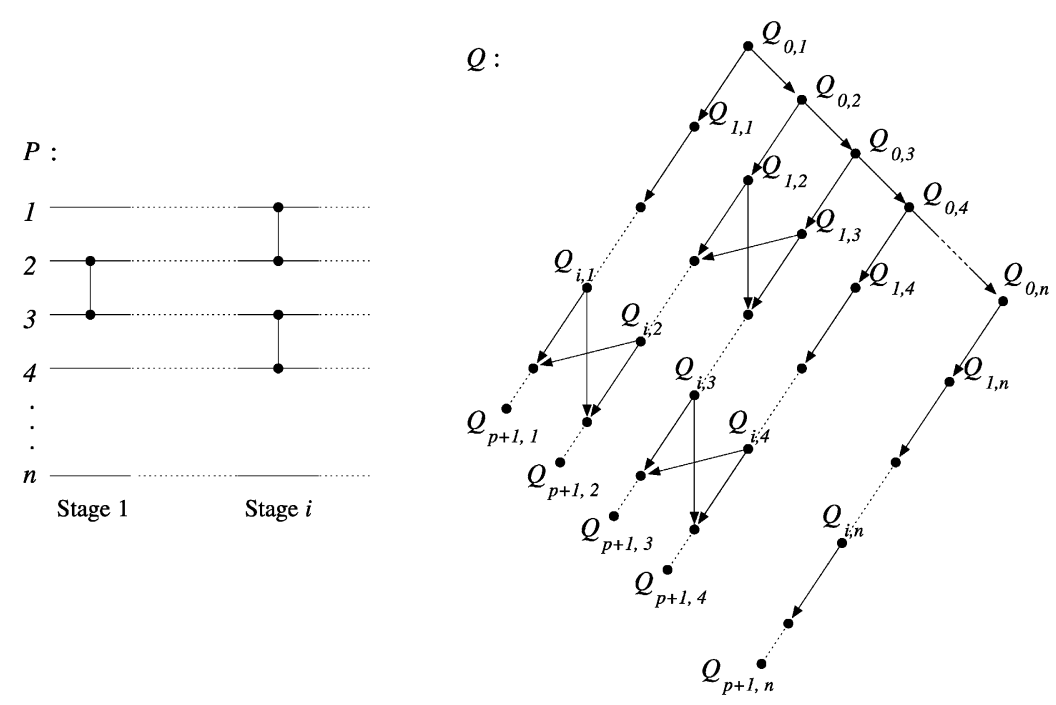

Fig. 2. The sorting network $P$ on the left yields a directed acyclic graph $Q$.

$(\{y, z\}, x)$ is an embedded subtree of $N$. The above argument can be repeated for any permutations $\pi_{y}$ and $\pi_{z}$ such that $\pi_{y}(y)=1, \pi_{y}(x)=2, \pi_{y}(z)=3$ and $\pi_{z}(z)=1, \pi_{z}(x)=2, \pi_{z}(y)=3$, respectively, to show that $(\{x, z\}, y)$ and $(\{x, y\}, z)$ are also consistent with $N$.

This yields:

Theorem 3. Given any set $L$ of $n$ leaf labels, a phylogenetic network consistent with all $3 \cdot\left(\begin{array}{l}n \\ 3\end{array}\right)$ rooted triplets whose leaf labels belong to $L$ can be constructed in $\mathrm{O}(s(n))$ time, where $s(n)$ is the time required to construct a sorting network for $n$ elements.

By employing, e.g., an AKS sorting network (see [2]), we obtain $s(n)=\mathrm{O}(n \log n)$ in Theorem 3 .

\section{Constructing all phylogenetic networks having one hybrid node with one attached leaf}

This section presents an algorithm called OneHybridLeaf for inferring the set of all phylogenetic networks having a single hybrid node attached to exactly one leaf (and having no other hybrid nodes) which are consistent with a given set $\mathcal{T}$ of rooted triplets. This algorithm is later used as a subroutine by the main algorithm in Section 4. OneHybridLeaf assumes that its given set $\mathcal{T}$ of rooted triplets is dense. We first note the following.

Lemma 4. Let $\mathcal{T}$ be a dense set of rooted triplets and let $L$ be the leaf set of $\mathcal{T}$. There is at most one rooted, unordered tree distinctly leaf labeled by $L$ which is consistent with $\mathcal{T}$. Furthermore, if such a tree $R$ exists then it must be binary.

Proof. Suppose there exist two unordered, distinctly leaf-labeled trees $R$ and $R^{\prime}$ consistent with $\mathcal{T}$ such that $R \neq R^{\prime}$. Then, for some $x, y, z \in L,(\{x, y\}, z)$ is consistent with $R$ while $(\{x, z\}, y)$ is consistent with $R^{\prime}$. Since $\mathcal{T}$ is dense, at least one of $(\{x, y\}, z),(\{x, z\}, y)$, and $(\{y, z\}, x)$ belongs to $\mathcal{T}$. This yields a contradiction in all cases because $R$ cannot be consistent with $(\{x, z\}, y)$ or $(\{y, z\}, x)$ and $R^{\prime}$ cannot be consistent with $(\{x, y\}, z)$ or $(\{y, z\}, x)$ since $R$ and $R^{\prime}$ are trees.

Next, suppose $R$ is not binary. Then $R$ has a node $u$ with degree greater than two. Let $x, y$, and $z$ be leaves from three different subtrees rooted at children of $u$. $\mathcal{T}$ is dense, so at least one of $(\{x, y\}, z),(\{x, z\}, y)$, and $(\{y, z\}, x)$ belongs to $\mathcal{T}$. But none of these three rooted triplets is consistent with $R$. Contradiction. 


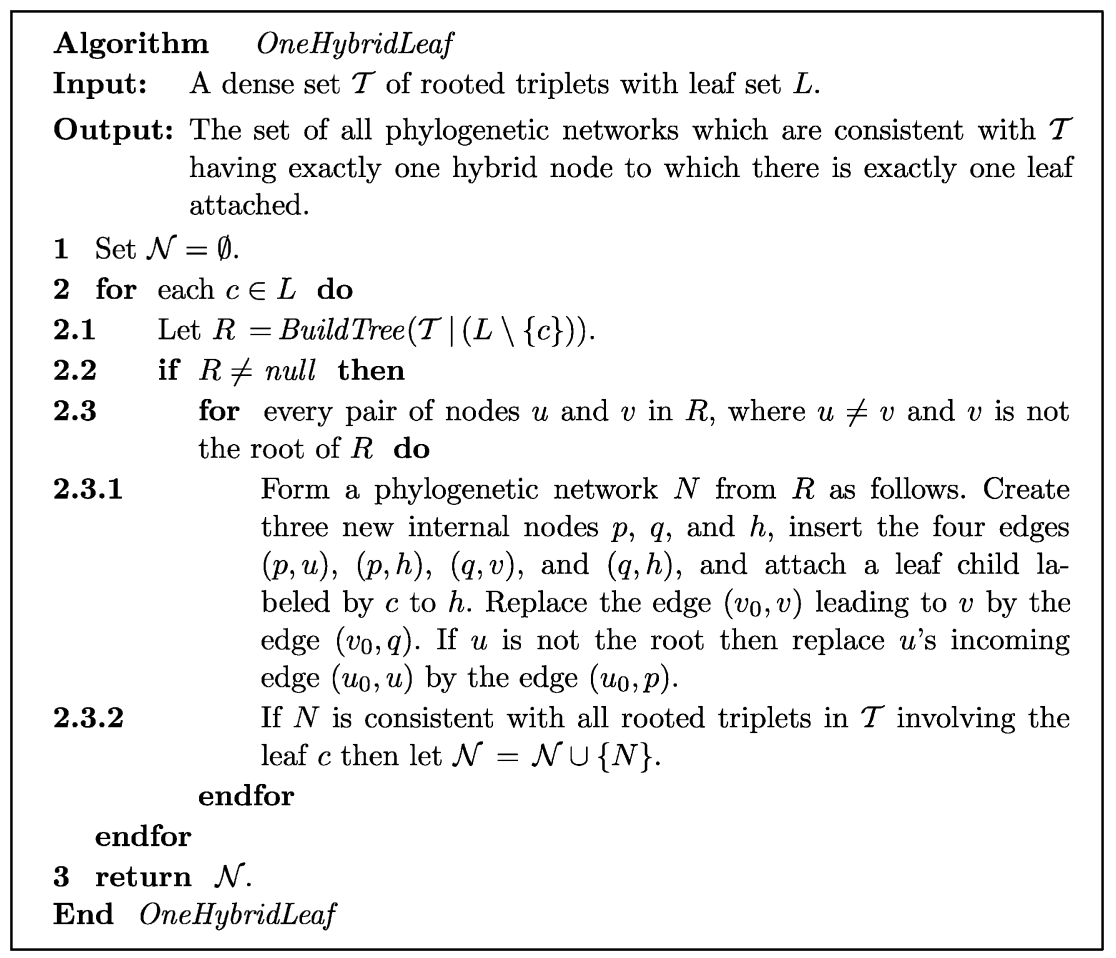

Fig. 3. Constructing phylogenetic networks with one hybrid node.

Lemma 5. Let $N$ be any phylogenetic network consistent with a set $\mathcal{T}$ of rooted triplets with leaf set $L$ such that $N$ has a hybrid node $h$ to which exactly one leaf $c$ is attached and $N$ has no other hybrid nodes. If $h$ and $c$ and all their incident edges are deleted and then, for every node with outdegree 1 and indegree less than 2, its outgoing edge is contracted, then the resulting graph is a binary tree consistent with $\mathcal{T} \mid(L \backslash\{c\})$.

Our algorithm OneHybridLeaf is shown in Fig. 3. It tests every $c \in L$ as the leaf attached to the hybrid node. For each such candidate $c$, it first calls a procedure BuildTree to obtain a binary tree $R$ which is consistent with all rooted triplets in $\mathcal{T}$ that do not involve the leaf $c$, if such a tree exists. ( $\mathcal{T}$ is dense, so the set $\mathcal{T} \mid(L \backslash\{c\})$ is also dense. Thus, Lemma 4 ensures that if $R$ exists then it is uniquely determined and binary.) Then, it tries all possible ways to obtain a phylogenetic network from $R$ by inserting a hybrid node $h$ attached to the leaf $c$, and keeps all resulting networks which are also consistent with the rest of $\mathcal{T}$. By Lemma 5, all valid phylogenetic networks will be found by OneHybridLeaf.

To implement the procedure BuildTree, we use the fast version of the algorithm of Aho et al. referred to in Lemma 1. If $L$ contains at least four elements then BuildTree $(\mathcal{T} \mid(L \backslash\{c\}))$ is the algorithm of Aho et al. applied to $\mathcal{T} \mid(L \backslash\{c\})$ (we may assume it returns null if it fails). For the case $|L|=3$, the set $\mathcal{T} \mid(L \backslash\{c\})$ is empty and we simply let BuildTree $(\mathcal{T} \mid(L \backslash\{c\}))$ return a tree with the two leaves in $L \backslash\{c\}$.

Lemma 6. The time complexity of Algorithm OneHybridLeaf is $\mathrm{O}\left(n^{5}\right)$.

Proof. Step 2 iterates Steps 2.1-2.3 $n$ times. In each iteration, Step 2.1 takes $\mathrm{O}\left(k+n^{2} \log n\right)$ time by Lemma 1. The inner for-loop (Step 2.3) considers $\mathrm{O}\left(n^{2}\right)$ pairs of nodes of $R$; for each such node pair, Step 2.3.1 takes O(1) time and Step 2.3.2 takes $\mathrm{O}\left(n^{2}\right)$ time. In total, Step 2.3 uses $\mathrm{O}\left(n^{2} \cdot\left(1+n^{2}\right)\right)=\mathrm{O}\left(n^{4}\right)$ time, so Step 2 takes $\mathrm{O}\left(n \cdot\left(k+n^{2} \log n+n^{4}\right)\right)$ time. Furthermore, $k=|\mathcal{T}|=\mathrm{O}\left(n^{3}\right)$. Thus, the total running time of OneHybridLeaf is $\mathrm{O}\left(n^{5}\right)$.

\section{Constructing a level-1 phylogenetic network}

Here, we present an algorithm called LevelOne for inferring a level-1 phylogenetic network (if one exists) consistent with a given dense set $\mathcal{T}$ of rooted triplets. The basic idea of our algorithm is to partition the leaf set $L$ of $\mathcal{T}$ into disjoint 


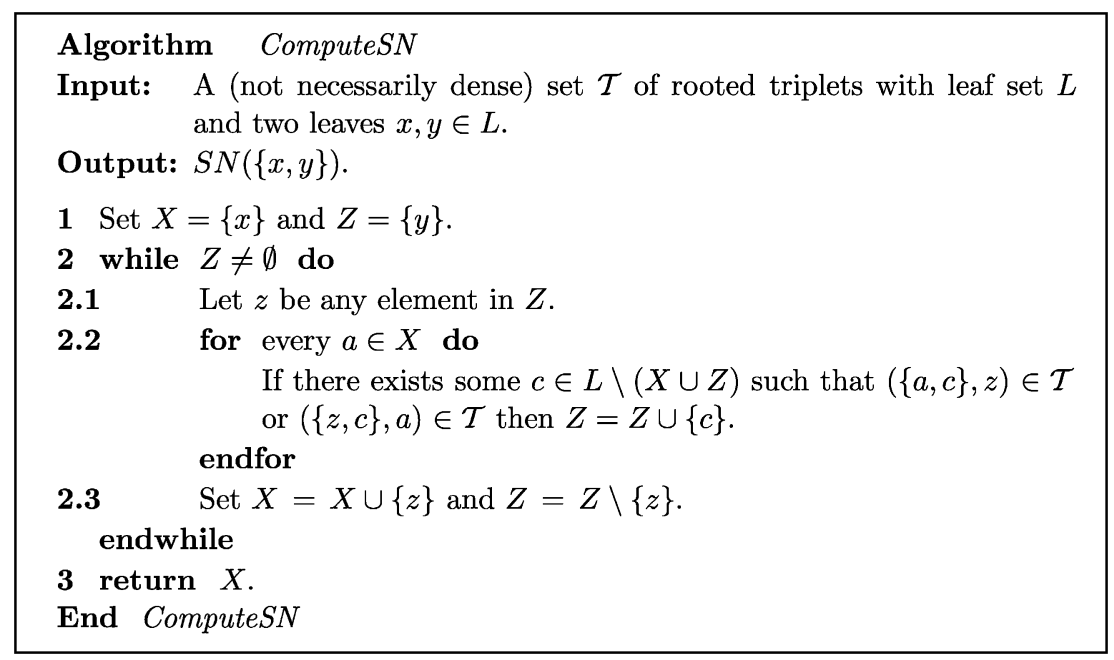

Fig. 4. Computing $S N(\{x, y\})$.

subsets which we call maximal $S N$-sets, run LevelOne recursively to construct a level-1 network for each such $S N$-set, and then apply Algorithm OneHybridLeaf from Section 3 to combine the computed networks for the $S N$-sets into one level-1 network.

We first introduce the concept of an $S N$-set. For any $X \subseteq L$, define the set $S N(X)$ recursively as $S N(X \cup\{c\})$ if there exist some $x_{1}, x_{2} \in X$ and $c \in L \backslash X$ such that $\left(\left\{x_{1}, c\right\}, x_{2}\right) \in \mathcal{T}$, and as $X$ otherwise. Intuitively, each $S N$-set is a subset of $L$ which will form the leaf set of a subnetwork of the final solution; hence the name " $S N$-set". Below, we study some properties of the $S N$-sets.

Lemma 7. $S N(\{x, y\})$ for any $x, y \in L$ is computable in $\mathrm{O}\left(n^{3}\right)$ time.

Proof. If $x=y$ then $S N(\{x, y\})=\{x\}$ can be obtained in $\mathrm{O}(1)$ time. If $x \neq y$ then $S N(\{x, y\})$ can be computed by Algorithm ComputeSN $(x, y)$ shown in Fig. 4. Initially, the algorithm sets $X=\{x\}$ and $Z=\{y\}$. Then, while $Z$ is nonempty, it selects any $z \in Z$, augments $Z$ with all leaves $c$ not already in $X \cup Z$ such that $(\{a, c\}, z)$ or $(\{z, c\}, a) \in \mathcal{T}$ for some $a \in X$, and finally removes $z$ from $Z$ and inserts $z$ into $X$. To analyze the time complexity of Algorithm ComputeSN, observe that one leaf is transferred from $Z$ to $X$ in each iteration of the while-loop and that a leaf which has been moved to $X$ can never be moved back to $Z$, so Steps 2.1-2.3 are iterated at most $n-1$ times. Inside the while-loop, the algorithm scans $\mathrm{O}\left(n^{2}\right)$ rooted triplets at most once to augment $Z$. The total running time of ComputeSN is therefore $\mathrm{O}\left(n^{3}\right)$.

Note that Lemma 7 holds even if $\mathcal{T}$ is not dense. However, if $\mathcal{T}$ is dense then the $S N$-sets have the following very important property.

Lemma 8. If $\mathcal{T}$ is dense then for any $A, B \subseteq L, S N(A) \cap S N(B)$ equals $\emptyset, S N(A)$, or $S N(B)$.

Proof. Suppose on the contrary that $z_{1}, z_{2} \in S N(A), z_{2}, z_{3} \in S N(B), z_{3} \notin S N(A)$, and $z_{1} \notin S N(B)$. Consider the rooted triplet on $z_{1}, z_{2}$, and $z_{3}$. Since $\mathcal{T}$ is dense, at least one of the following three cases must occur:

- Case 1: $\left(\left\{z_{2}, z_{3}\right\}, z_{1}\right) \in \mathcal{T}$. Then, by definition, $z_{3} \in S N(A)$.

- Case 2: $\left(\left\{z_{1}, z_{3}\right\}, z_{2}\right) \in \mathcal{T}$. Then, by definition, $z_{3} \in S N(A)$.

- Case 3: $\left(\left\{z_{1}, z_{2}\right\}, z_{3}\right) \in \mathcal{T}$. Then, by definition, $z_{1} \in \operatorname{SN}(B)$.

In each of the three cases, we have a contradiction. Thus, the lemma follows.

In particular, Lemma 8 holds for all subsets of $L$ of cardinality one or two. 

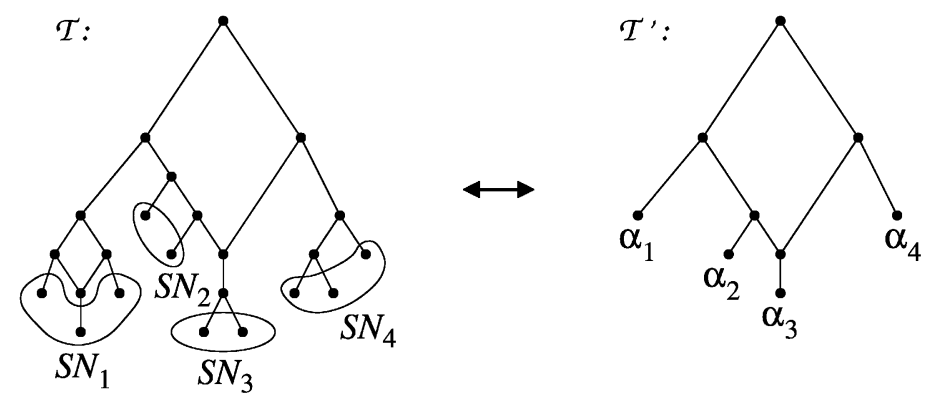

Fig. 5. Illustrating the correspondence between $\mathcal{T}$ and $\mathcal{T}^{\prime}$. Each set $S N_{i} \in \mathcal{S N}$ in a level-1 phylogenetic network consistent with $\mathcal{T}$ corresponds to a leaf $\alpha_{i}$ in a phylogenetic network consistent with $\mathcal{T}^{\prime}$ having at most one hybrid node (see the proof of Lemma 9), and vice versa (see Lemma 10).

From here on, we assume $\mathcal{T}$ is dense. For any $x_{1}, x_{2} \in L$ (possibly with $x_{1}=x_{2}$ ), $S N\left(\left\{x_{1}, x_{2}\right\}\right.$ ) is called trivial if $S N\left(\left\{x_{1}, x_{2}\right\}\right)=L$, and $S N\left(\left\{x_{1}, x_{2}\right\}\right)$ is called maximal if it is nontrivial and not a proper subset of any nontrivial $S N\left(\left\{y_{1}, y_{2}\right\}\right)$, where $y_{1}, y_{2} \in L$. Let $\mathcal{S N}$ be the set of all maximal $S N$-sets of the form $S N\left(\left\{x_{1}, x_{2}\right\}\right)$, where possibly $x_{1}=x_{2}$. Since $\mathcal{T}$ is dense, $\mathcal{S N}$ forms a partition of the set $L$ by Lemma 8.Furthermore, $\mathcal{S N}$ is uniquely determined. Write $\mathcal{S N}=\left\{S N_{1}, S N_{2}, \ldots, S N_{q}\right\}$ and introduce $q$ new symbols $\alpha_{1}, \alpha_{2}, \ldots, \alpha_{q}$. (Observe that $q \geqslant 2$ if $|L| \geqslant 2$.) We define a function $f$ as follows. For every $x \in L$, let $f(x)=\alpha_{i}$ if $x \in S N_{i}$. Let $\mathcal{T}^{\prime}$ be the set $\{(\{f(x), f(y)\}, f(z))$ : $(\{x, y\}, z) \in \mathcal{T}$ and $f(x), f(y), f(z)$ all differ $\}$.

The next two lemmas imply that $\mathcal{T}$ is consistent with a level-1 phylogenetic network if and only if $\mathcal{T}^{\prime}$ is consistent with a phylogenetic network with at most one hybrid node. See Fig. 5.

Lemma 9. Suppose $\mathcal{T}$ is consistent with a level-1 phylogenetic network. If $q=2$ then the tree distinctly leaf labeled by $\alpha_{1}$ and $\alpha_{2}$ is consistent with $\mathcal{T}^{\prime}$. If $q \geqslant 3$ then there exists a phylogenetic network having a single hybrid node attached to exactly one leaf (and having no other hybrid nodes) that is consistent with $\mathcal{T}^{\prime}$.

Proof. If $q=2$ then $\mathcal{T}^{\prime}$ is empty, and so the tree distinctly leaf labeled by $\alpha_{1}$ and $\alpha_{2}$ is always consistent with $\mathcal{T}^{\prime}$. Below, we consider the case $q \geqslant 3$.

Let $M$ be any level-1 phylogenetic network with leaf set $L$ that is consistent with $\mathcal{T}$. First, observe that there must exist two paths $p_{1}$ and $p_{2}$ from the two children of the root $r$ to a hybrid node in $M$. (To see this, suppose the opposite holds and let $A$ and $B$ be the disjoint sets of leaves in the two subnetworks rooted at the children of $r$. For each $S N_{i} \in \mathcal{S N}$, either $S N_{i} \subseteq A$ or $S N_{i} \subseteq B$ because $\mathcal{T}$ is dense and because $S N_{i}$ is nontrivial. Since $q \geqslant 3$, there exist $i, j, k$ where $i, j, k$ differ such that both $S N_{i}$ and $S N_{j}$ are subsets of one of $A$ and $B$, and $S N_{k}$ is a subset of the other. Both $S N_{i}$ and $S N_{j}$ are proper subsets of $S N(\{x, y\})$ for any $x \in S N_{i}$ and $y \in S N_{j}$ by Lemma 8. However, $S N(\{x, y\})$ contains no leaves from $S N_{k}$ and is therefore nontrivial. This implies that $S N_{i}$ and $S N_{j}$ are not maximal, which gives us a contradiction.)

For any node $u$ in $M$, denote the subnetwork of $M$ rooted at $u$ by $M[u]$. If $u$ is not located on $p_{1}$ or $p_{2}$ but a parent of $u$ is, then $u$ is called a side node in $M$. If $u$ is a side node then $u$ has only one parent, which we denote by $P(u)$. For each side node $u$ in $M$, it holds that $M[u]$ can contain leaves from one $S N_{i} \in \mathcal{S N}$ only (if $M[u]$ contains two leaves $x \in S N_{i}$ and $y \in S N_{j}$ where $i \neq j$ then $S N_{i} \subsetneq S N(\{x, y\})$ and $S N_{j} \subsetneq S N(\{x, y\})$ by Lemma 8 , and moreover, since $u$ is a side node, we have $S N(\{x, y\}) \neq L$ and thus $S N_{i}$ and $S N_{j}$ are not maximal, which is a contradiction). Next, note that if $M[u]$ and $M[v]$ for two side nodes $u, v$ in $M$ contain leaves from the same $S N_{i} \in \mathcal{S N}$ then $P(u)$ and $P(v)$ must both belong to $p_{1}$ or both belong to $p_{2}$ (otherwise, $S N_{i}=L$, which is impossible). Furthermore, for every side node $w$ such that $P(w)$ lies on the path between $P(u)$ and $P(v)$, the leaves in $M[w]$ must also belong to the same $S N_{i}$. This means that the parents of all side nodes in $M$ that have descendant leaves from the same $S N_{i} \in \mathcal{S N}$ are consecutively ordered along either $p_{1}$ or $p_{2}$. For each $S N_{i} \in \mathcal{S N}$, we can therefore concatenate all subnetworks that are rooted at a side node in $M$ and whose leaves belong to $S N_{i}$ to obtain a phylogenetic network $M^{*}$ consistent with $\mathcal{T}$ such that each subnetwork $M_{i}^{*}$ rooted at a side node in $M^{*}$ is bijectively leaf labeled by one $S N_{i} \in \mathcal{S N}$. Finally, by replacing each such $M_{i}^{*}$ with a leaf labeled by $\alpha_{i}$, we obtain a phylogenetic network consistent with $\mathcal{T}^{\prime}$ having one hybrid node. 


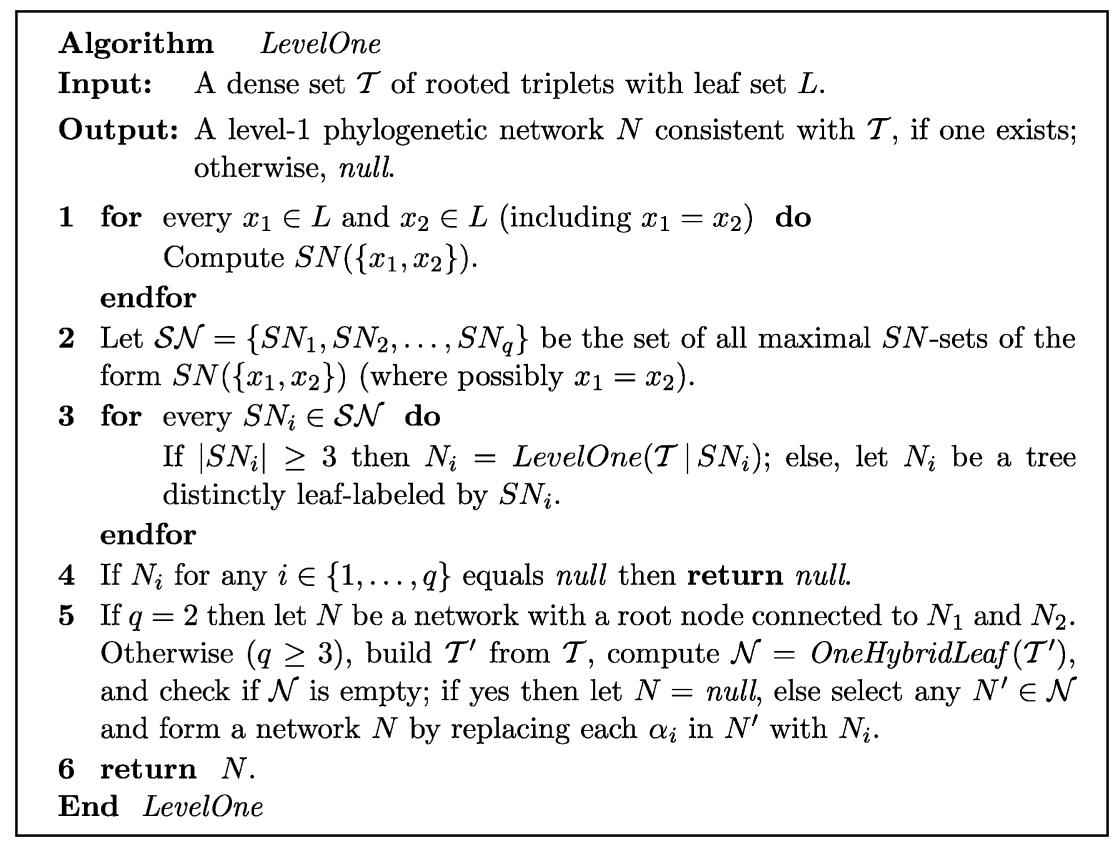

Fig. 6. Constructing a level-1 phylogenetic network.

Lemma 10. Suppose $\mathcal{T}^{\prime}$ is consistent with a level-1 phylogenetic network $N^{\prime}$ with leaf set $\left\{\alpha_{1}, \ldots, \alpha_{q}\right\}$. Let $N$ be a level-1 network obtained from $N^{\prime}$ by replacing each $\alpha_{i}$ by a level-1 network $N_{i}$ with leaf set $S N_{i}$ consistent with $\mathcal{T} \mid S N_{i}$. Then $N$ is consistent with $\mathcal{T}$.

Proof. Let $t$ be any rooted triplet in $\mathcal{T}$ and write $t=(\{x, y\}, z)$. If $x \in S N_{i}, y \in S N_{j}$, and $z \in S N_{k}$, where $i, j, k$ all differ, then $t$ is consistent with $N$ (otherwise, $t^{\prime}=(\{f(x), f(y)\}, f(z))=\left(\left\{\alpha_{i}, \alpha_{j}\right\}, \alpha_{k}\right)$ cannot be consistent with $N^{\prime}$ which is a contradiction since $t^{\prime} \in \mathcal{T}^{\prime}$ ). If $x, y \in S N_{i}$ and $z \in S N_{j}$ with $i \neq j$ then $t$ is consistent with $N$ by the construction of $N$. The case $x, z \in S N_{i}$ and $y \in S N_{j}$ (or symmetrically, $y, z \in S N_{i}$ and $x \in S N_{j}$ ) with $i \neq j$ is not possible since $x, z \in S N_{i}$ would imply $y \in S N_{i}$. If $x, y, z$ belong to the same $S N_{i}$ then $t$ is consistent with $N_{i}$ and therefore with $N$. In all cases, $t$ is consistent with $N$.

Our main algorithm LevelOne is listed in Fig. 6. Its correctness follows from Lemmas 9 and 10.

Theorem 11. When $\mathcal{T}$ is dense, we can determine if there exists a level-1 phylogenetic network consistent with $\mathcal{T}$, and if so construct one, in $\mathrm{O}\left(n^{6}\right)$ time.

Proof. Apply Algorithm LevelOne to $\mathcal{T}$. For any $L^{\prime} \subseteq L$, let $g\left(L^{\prime}\right)$ be the running time of LevelOne $\left(\mathcal{T} \mid L^{\prime}\right)$. In Step 1 of the algorithm, we compute $S N\left(\left\{x_{1}, x_{2}\right\}\right)$ for the $n^{2}$ pairs $\left(x_{1}, x_{2}\right)$ in $L \times L$. By Lemma 7, Step 1 takes $\mathrm{O}\left(n^{5}\right)$ time. Step 2can be performed in $\mathrm{O}\left(n^{3}\right)$ time, and Step 3takes $\sum_{S N_{i} \in \mathcal{S N}} g\left(S N_{i}\right)$ time. Step 5can be done in $\mathrm{O}\left(n^{5}\right)$ time according to Lemma 6. In total, we have $g(L)=\sum_{S N_{i} \in \mathcal{S N}} g\left(S N_{i}\right)+\mathrm{O}\left(n^{5}\right)$. Since all sets in $\mathcal{S N}$ are disjoint, $g(L)=\mathrm{O}\left(n^{6}\right)$.

Algorithm LevelOne can be modified to return all level-1 phylogenetic networks consistent with $\mathcal{T}$ by utilizing all the possible topologies returned by OneHybridLeaf. However, the running time may then become exponential since some inputs are consistent with an exponential number of different level-1 networks. (At each recursion level, although the partition of the leaves into $\mathcal{S N}$ is unique when the input is dense, there may be more than one way to merge the recursively computed subnetworks for the $S N$-sets into a valid network.) 


\section{Concluding remarks}

This paper presents a polynomial-time algorithm for inferring a level-1 phylogenetic network from a dense set of rooted triplets. In the future, we plan to further improve the time complexity of our main algorithm and to investigate the computational complexity of the problem when $\mathcal{T}$ is not dense. Also, we would like to know if it is possible to construct a level- $f$ phylogenetic network from a dense set of rooted triplets in polynomial time for any constant $f>1$. Finally, since a set of rooted triplets based on experimental data might not be consistent with any level-1 phylogenetic network, it would be interesting to consider the optimization version of the problem where the objective is to construct a level-1 phylogenetic network consistent with as many rooted triplets from $\mathcal{T}$ as possible (for the rooted tree case, this maximization problem was studied in [3,8,9,14,24]; see also [15]).

\section{Note added in proof}

The results presented in this article have recently been extended and strengthened in [25].

\section{References}

[1] A.V. Aho, Y. Sagiv, T.G. Szymanski, J.D. Ullman, Inferring a tree from lowest common ancestors with an application to the optimization of relational expressions, SIAM J. Comput. 10 (3) (1981) 405-421.

[2] M. Ajtai, J. Komlós, E. Szemerédi, An $O(n \log n)$ sorting network, in: Proc. 15th Annu. ACM Symp. on the Theory of Computing (STOC'83), 1983, pp. 1-9.

[3] D. Bryant. Building trees, hunting for trees, and comparing trees: theory and methods in phylogenetic analysis, Ph.D. Thesis, University of Canterbury, Christchurch, New Zealand, 1997.

[4] D. Bryant, V. Moulton, Neighbor-net: an agglomerative method for the construction of phylogenetic networks, Mol. Biol. Evol. 21 (2) (2004) $255-265$.

[5] B. Chor, M. Hendy, D. Penny, Analytic solutions for three-taxon $\mathrm{ML}_{M C}$ trees with variable rates across sites, in: Proc. of the First Workshop on Algorithms in Bioinformatics (WABI 2001), Lecture Notes in Computer Science, Vol. 2149, Springer, Berlin, 2001, pp. $204-213$.

[6] C. Choy, J. Jansson, K. Sadakane, W.-K. Sung, Computing the maximum agreement of phylogenetic networks, Theoret. Comput. Sci. 335 (1) (2005) 93-107.

[7] T. Cormen, C. Leiserson, R. Rivest, Introduction to Algorithms, The MIT Press, MA, 1990.

[8] L. Ga̧sieniec, J. Jansson, A. Lingas, A. Östlin, Inferring ordered trees from local constraints, in: Proc. of Computing: the Fourth Australasian Theory Symposium (CATS'98), Australian Computer Science Communications, Vol. 20(3), Springer, Singapore, 1998, pp. 67-76.

[9] L. Gąsieniec, J. Jansson, A. Lingas, A. Östlin, On the complexity of constructing evolutionary trees, J. Combin. Optim. 3 (2-3) (1999) 183-197.

[10] D. Gusfield, S. Eddhu, C. Langley, Efficient reconstruction of phylogenetic networks with constrained recombination, in: Proc. of the Computational Systems Bioinformatics Conference (CSB2003), 2003, pp. 363-374.

[11] J. Hein, Reconstructing evolution of sequences subject to recombination using parsimony, Math. Biosci. 98 (2) (1990) 85-200.

[12] M.R. Henzinger, V. King, T. Warnow, Constructing a tree from homeomorphic subtrees, with applications to computational evolutionary biology, Algorithmica 24 (1) (1999) 1-13.

[13] J. Holm, K. de Lichtenberg, M. Thorup, Poly-logarithmic deterministic fully-dynamic algorithms for connectivity minimum spanning tree, 2-edge, and biconnectivity, J. ACM 48 (4) (2001) 723-760.

[14] J. Jansson, On the complexity of inferring rooted evolutionary trees, in: Proc. of the Brazilian Symp. on Graphs, Algorithms, and Combinatorics (GRACO 2001), Electronic Notes in Discrete Mathematics, Vol. 7, Elsevier, Amsterdam, 2001, pp. 121-125.

[15] J. Jansson, J.H.-K. Ng, K. Sadakane, W.-K. Sung, Rooted maximum agreement supertrees. Algorithmica 43 (4) (2005) $293-307$.

[16] T. Jiang, P. Kearney, M. Li, A polynomial time approximation scheme for inferring evolutionary trees from quartet topologies and its application, SIAM J. Comput. 30 (6) (2001) 1942-1961.

[17] S. Kannan, E. Lawler, T. Warnow, Determining the evolutionary tree using experiments, J. Algorithms 21 (1) (1996) 26-50.

[18] P. Kearney, Phylogenetics and the quartet method, in: T. Jiang, Y. Xu, M.Q. Zhang (Eds.), Current Topics in Computational Molecular Biology, The MIT Press, MA, 2002, pp. 111-133.

[19] L. Nakhleh, J. Sun, T. Warnow, C.R. Linder, B.M.E. Moret, A. Tholse, Towards the development of computational tools for evaluating phylogenetic reconstruction methods, in: Proc. Eighth Pacific Symp. on Biocomputing (PSB 2003), 2003, pp. 315-326.

[20] L. Nakhleh, T. Warnow, C.R. Linder, Reconstructing reticulate evolution in species-theory and practice, in: Proc. Eighth Annu. Internat. Conf. on Research in Computational Molecular Biology (RECOMB 2004), 2004, pp. 337-346.

[21] D. Posada, K.A. Crandall, Intraspecific gene genealogies: trees grafting into networks, TRENDS Ecol. Evol. 16 (1) (2001) $37-45$.

[22] M. Steel, The complexity of reconstructing trees from qualitative characters and subtrees, J. Classification 9 (1) (1992) $91-116$.

[23] L. Wang, K. Zhang, L. Zhang, Perfect phylogenetic networks with recombination, J. Comput. Biol. 8 (1) (2001) 69-78.

[24] B.Y. Wu, Constructing the maximum consensus tree from rooted triples, J. Combin. Optim. 8 (2004) 29-39.

[25] J. Jansson, N.B. Nguyen, W.-K. Sung, Rooted maximum agreement supertrees, Algorithmica. 43 (4) (2005) 293-307. 